\title{
Industrie des matières premières et responsabilité sociale d'entreprise en Afrique : réflexion sur le cadre juridique international
}

\author{
Par Bahellaby Timothée ${ }^{1}$
}

\section{Résumé}

Depuis la décennie septante, les Etats africains luttent pour une industrie des matières premières susceptible d'accélérer le progrès socio-économique dans le continent et plus respectueuse des droits fondamentaux des travailleurs et propriétaires terriens. Dès lors, cet effort est freiné par des pesanteurs liées notamment aux comportements des multinationales capitalistes dont la plupart violent les droits des peuples, contribuent à institutionnaliser la corruption, mènent des activités qui polluent et dégradent l'environnement et hypothèquent ainsi les perspectives du développement durable. Face à cette situation, la communauté internationale a, tant bien que mal, réagi en instituant des mécanismes juridiques en vue d'encadrer les activités des multinationales sur les territoires étatiques, surtout dans les industries des matières premières où les abus paraissent plus éloquents. Cependant, ces mécanismes ont une portée facultative car ne pouvant contraindre ni l'Etat ni l'entreprise multinationale, dont la politique y contrevient. Dans ces conditions, l'Afrique en sort marginalisée car ne sachant porter sa voix plus haute en vue d'obtenir des engagements plus fermes des multinationales dont la position à la fois d'acheteur et de vendeur ne cesse de préjudicier l'Afrique déjà fragilisée par des crises multiformes. En revanche, le régionalisme serait l'une des pistes de sortie ; car il permettrait non seulement de conclure des accords commerciaux plus contraignants, d'élargir le marché régional susceptible d'absorber les ressources produites mais aussi de conclure des accords commerciaux plus contraignants et mutuellement avantageux avec les partenaires commerciaux, en privilégiant la transformation des matières premières sur le territoire africain, nécessaire à l'essor des économies nationale et régionale. Et la vision minière pour l'Afrique s'avère ainsi le nouveau paradigme dans l'effort de commerce équitable ou mieux de justice économique dans les industries minières. Dans cet engrenage, l'Etat demeure un acteur incontournable dans la préservation des intérêts nationaux et surtout des peuples autochtones face aux appétits dévastateurs des multinationales capitalistes. Un leadership visionnaire et responsable en constitue la condition sine qua non pour une responsabilité sociale d'entreprise effective en Afrique.

1 Assitant et Doctorant à la Faculté de Droit de l'UNIKIN/ Avocat à la Cour. Domaines de recherche: droit communautaire comparé ; développement durable, gestion des ressources naturelles et justice économique ; criminalité transnationale organisée. E-mail : grectim@gmail.com; Tel.: + 2438564 21064 


\section{INTRODUCTION}

L'Afrique est généralement décrite comme l'un des continents qui, outre une population croissante, à prédominance jeune, de vastes terres et des sols fertiles, regorge de ressources naturelles ou mieux minérales considérables mais dont les populations demeurent les plus pauvres de la planète. L'Afrique possède environ $12 \%$ des réserves mondiales de pétrole, $42 \%$ des réserves d'or, entre 80 et $90 \%$ des réserves de métaux du groupe du chrome et du platine, $60 \%$ des terres arables et de vastes ressources en bois ${ }^{2}$ qui peuvent être mises à profit pour promouvoir l'industrialisation et la transformation économique structurelle par des stratégies de création de valeur ajoutée dans tous les secteurs productifs (agriculture et services).

Dès lors, les importantes ressources naturelles de l'Afrique sont extraites et exportées sous forme de produits bruts et non de produits finis ou industrialisés ; c'est là une sérieuse occasion manquée pour un développement durable. En outre, certaines des ressources sont non renouvelables, et leur exploitation donne lieu en général à de faibles liens avec le reste de l'économie et, partant, à une plus faible contribution au PIB que cela n'aurait pu être le cas. $^{3}$

Forts de richesses abondantes et de la demande mondiale croissante de matières premières, les gouvernements africains établissent actuellement de nouveaux partenariats, s'emploient à acquérir de la technologie, à accroître les investissements dans les infrastructures, et dans les industries des matières premières ou produits de base.

En effet, la promotion d'une industrialisation fondée sur les produits de base pourrait offrir un puissant outil aux pays africains pour s'attaquer au monde de la finance internationale. Cette industrialisation ne devrait non seulement s'appuyer sur les minerais mais aussi sur d'autres produits de base notamment agricoles. Cependant, l'effort de diversification de l'industrie des matières premières est freiné par certains pesanteurs, notamment l'exportation des matières premières à l'état brut. Ce phénomène entrave la création d'emplois et la distribution des rentes tirées de leur exploitation. En dépit de maintes initiatives des pouvoirs publics africains de mettre en valeur et faire profiter les minerais aux peuples autochtones, des principaux problèmes qui se posent à l'Afrique en matière de mise en valeur de ses ressources naturelles demeurent toujours d'actualité : l'absence de données d'information sur les gisements de ressources naturelles de vastes zones inexplorées...; l'insuffisance des moyens requis (capitaux, compétences et technologie) pour leur mise en valeur; une forte dépendance à l'égard des sociétés transnationales qui n'exploitent que quelques res-

2 Voir CEA et UA, Tirer le plus grand profit des produits de base africains: l'industrialisation au service de la croissance, de l'emploi et de la transformation économique, Rapport économique sur l'Afrique 2013, pp. 8-10.

3 CEA et UA, L'industrialisation au service de l'émergence de l'Afrique, Document de synthèse de la Sixième réunion conjointe de la Conférence des ministres africains des finances, de la planification et du développement économique de la Commission économique pour l'Afrique et de la Conférence des ministres de l'économie et des finances de l'Union africaine, Abidjan (Côte d'Ivoire) 25 et 26 mars 2013, p. 11. 
sources naturelles africaines pour produire les matières premières nécessaires aux pays développés ${ }^{4}$. A ceux-ci se juxtaposent le partage inégal de la valeur ajoutée issue de l'exploitation des ressources naturelles des États membres; un niveau de développement et une exploitation extrêmement faibles des ressources naturelles délaissées par les sociétés transnationales; et une contribution dramatiquement faible des richesses naturelles au développement socio-économique ${ }^{5}$.

«Une trajectoire de croissance plus durable, inclusive et équitable dans les économies exportatrices de produits de base passe par la possibilité d'établissement de liens en amont et en aval pour la production de ces produits $\rangle^{6}$. L'une des conséquences de ces pesanteurs est qu'en dépit de la croissance de l'Afrique qui excède la moyenne mondiale au cours de cette décennie, celle-ci elle ne se traduit pas encore par une réduction correspondante de la pauvreté à un moment où le niveau de pauvreté chutait dans d'autres parties du monde; ce qui donne une image biaisée de la réduction de la pauvreté dans le monde. De même, la dispersion de la production à l'échelle mondiale a entrainé des avantages inégaux qui ont le plus profité aux économies de l'Asie de l'Est et du Sud-Est, en particulier à la Chine ${ }^{7}$.

Plus d'un estiment que l'absence d'un mécanisme conventionnel de portée synallagmatique et contraignante à l'égard des entités étatiques et, surtout, privées œuvrant dans les industries extractives, justifierait les faiblesses du système industriel africain dans ce domaine. Ceci est d'autant plus vrai que la répartition des dividendes découlant des industries extractives demeure encore inéquitable dans la mesure où les Etats importateurs des matières brutes tirent largement profit au détriment de leurs pairs africains producteurs ${ }^{8}$. En contrôlant l'essentiel des marchés des matières premières et des biens d'équipement, en ayant le monopole des capitaux et des services, les grandes puissances et les multinationales néolibéralistes fixent les prix des matières de base exportées par les pays en voie de développement ainsi que des services qu'ils leur fournissent. Cet ordre est aussi périmé et injuste que l'ordre colonial dont il tire son origine et sa substance. Dans ces conditions, le commerce équitable ou la justice économique dans le contexte de la mondialisation est loin d'être une réalité pour l'Afrique.

Selon Smail Khannas, "les contraintes actuelles du continent africain notamment l'endettement, le chômage, la faible productivité, la croissance démographique et l'autorité de l'Etat déficitaire (...), particulièrement en matière économique, « (...) semblent constituer

4 LEVACHER, C., Les sociétés transnationales minières face au droit des peuples autochtones. Quels acteurs, pour quels enjeux?, GITPA, Septembre 2012, p. 12.

5 Voir CEA et UA, Tirer le plus grand profit des produits de base africains...op.cit., p. 10.

6 CEA et UA, op.cit. En réalité, la plupart de pays africains ne sont pas producteurs mais plutôt pourvoyeurs des matières premières, puisque ce sont les multinationales privées et essentiellement étrangères qui exploitent et produisent ces dernières.

7 Idem.

8 Voir CEA et UA, Tirer le plus grand profit des produits de base africains... op.cit., p. 9. 
des freins majeurs à tout processus de développement $»^{9}$. Aussi, dans leur processus d'industrialisation, des politiques de transfert technologique développées par certains pays africains dans la constitution de sociétés mixtes et d'achats de brevets étaient fortement compromises par l'insuffisance d'un environnement humain et matériel susceptibles d'assimiler et surtout de maintenir et de reproduire la technologie ainsi que les biens d'équipement. De plus, l'on note des difficultés d'absorption de la technologie importée exacerbées par des pratiques protectionnistes, parfois informelles, et des contrats tendant à institutionnaliser un rapport de forces inégal.

Dès lors, comment l'Afrique peut-il tirer le plus grand profit de ses produits de base? Dans le contexte socio-économique qui les caractérise, comment les Etats africains peuvent imaginer des accords bi et multilatéraux susceptibles de booster des processus d'industrialisation des matières premières au bénéfice de la transformation économique et de l'emploi ?

D'ores et déjà, il est impérieux de mettre un terme au contrôle des mécanismes de transfert des richesses des pays pauvres et principalement du système de fixation des prix des matières de base par les monopoles internationaux et les firmes multinationales. En tant que porte-étendard de l'Afrique, l'UA devrait instituer des mécanismes juridiques contraignants en la matière dont la violation engagerait la responsabilité des auteurs.

En Afrique, « l'on a pendant longtemps considéré comme allant de soi qu'il y ait toujours des gagnants et des perdants dans l'exploitation minière» ${ }^{10}$. Il est vrai que certaines parties concernées, notamment les communautés locales, et peut-être même les États, sont loin d'y trouver leur compte, comme le montrent amplement l'étendue de la pauvreté et le grave déficit infrastructurel de l'Afrique, ainsi que la faible voix du continent dans les négociations portant sur les contrats miniers.

En revanche, la Vision africaine des mines adoptée par les chefs d'État et de gouvernement en février 2009, s'avère un effort de plus pour inverser cette situation. Elle préconise une exploitation transparente, équitable et optimale des ressources minérales en tant que fondement d'une croissance durable et d'un développement socio-économique généralisé en Afrique. Au cœur de la Vision se trouve le principe d'un État développementiste ${ }^{11}$ intégrant le secteur minier dans des processus plus larges de développement. L'émergence du paradigme de marché gagnant-gagnant (win-win) qui admet un gain réciproque et équitable pour l'Afrique et ces partenaires commerciaux, s'inscrit dans cette vision. Cependant, seuls, les accords internationaux ne sauraient répondre aux énormes attentes sociales si la gouvernance interne demeure asymétrique. L'une des pistes résiderait dans des politiques indus-

9 SMAIL KHENNAS, «Préface », in SMAIL KHENNAS (dir.), Industrialisation, ressources minières et énergie en Afrique, Dakar, CODESRIA, 1993.

10 CEA et UA, op.cit.

11 L'expression est tirée de la note d'information sur la vision minière pour l'Afrique, des membres du Forum Afrique-Canada du CCCI et du Réseau canadien pour la reddition de compte des entreprises, voir supra. 
trielles axées sur la transformation et le raffinage des matières premières sur le territoire de l'Etat pourvoyeur : responsabilité qui relève des gouvernants. ${ }^{12}$

Toutefois, le défi de la pauvreté est devenu la priorité à laquelle il faut absolument faire face si l'on veut éviter des conflagrations internationales incontrôlables et de plus en plus dramatiques. La production et l'exportation des matières premières à l'état brut équivalent à un abandon de recettes énormes du fait de la non-création de valeur ajoutée, à l'exportation d'emplois vers les pays qui peuvent ajouter de la valeur aux produits et à l'exposition à de hauts risques inhérents à la dépendance à l'égard des produits non renouvelables et des fluctuations de la demande et des prix des produits de base. ${ }^{13}$

Malgré les difficultés actuelles, l'Afrique dispose des avantages comparatifs dans tous les domaines, notamment les matières premières abondantes, le coût relativement faible de la main d'œuvre et un large marché régional et compétitif à exploiter. Ces atouts ne peuvent être lucratifs que si les règles qui les encadrent sont négociées de manière à produire les effets mutuellement avantageux à l'égard de deux parties aux termes de l'article 2 de la Convention de vienne sur le droit des traités.

12 Le terme de matières premières est vague; il ne doit pas être confondu avec des termes voisins souvent employés indifféremment dans le langage courant et même dans le vocabulaire diplomatique. Il convient de retenir une définition large qui recouvre tous les produits autres que les produits semi-finis ou manufacturés. Les caractères techniques, le régime économique, l'importance sociale des diverses matières premières exportées sont très variables : aussi plusieurs typologies des matières premières sont-elles proposées. On peut distinguer par exemple les minéraux, les combustibles, les matières premières agricoles destinées à l'industrie et les matières premières alimentaires. D'autres classements peuvent être effectués à divers points de vue. Pour plus de détails sur ce distinguo, voir infra, voir TOUSCOZ J., « La coopération internationale et les matières premières exportées par les pays du Tiers Monde : les conditions d'un échange égal », Tiers-monde, 1976, tome $17 \mathrm{n}^{\circ} 66$, les produits de base et la politique internationale, pp. 539-560, disponible en ligne sur http://www.persee.fr/web/revue/home/prescript/article/tiers_0040-7356_1976_num_17_6 6_2648, consulté le 14 septembre 2014. Dans le cadre de cette étude, les matières premières peuvent s'entendre dans une acception plus large. Par ailleurs, l'industrie peut s'entendre ici comme le secteur de production mécanisée et concentrée produisant des biens et services ou encore comme le secteur d'activité organisé produisant des richesses ou des services mais surtout les transforme en produits finis.

13 TATOU, R., Europe outre-mer, avril 1974, n531, p. 9-11. 
Dans une perspective " évaluative et prescriptive» ${ }^{14}$, l'analyse portera essentiellement sur la critique des règles et principes à observer par les entreprises ${ }^{15}$ et les Etats pour une industrie respectueuse des droits de l'homme, des normes environnementales et de lutte contre la corruption : la responsabilité sociale d'entreprise. De ce fait, les aspects purement procéduraux en termes de création et de fonctionnement des entreprises au regard du droit des affaires ne seront pas abordés dans le cadre de cette étude. Il s'agit plutôt de procéder à l'exercice de repérage et de décryptage des normes internationales relatives à l'industrie des matières premières. Avant cela, un regard sur l'évolution des tentatives normatives de la $\mathrm{RSE}^{16}$ s'avère utile. Le développement durable étant la finalité, quelques initiatives et politiques de développement industriel en Afrique seront épinglées et jonchées d'une critique susceptible de déboucher sur des perspectives d'industrialisation des matières premières en Afrique répondant à la RSE.

\section{La responsabilité sociale d'entreprise : regard rétrospectif des efforts d'encadrement juridique international}

Au cours de la décennie septante, les pays dits du tiers monde avaient formulé des critiques à l'endroit du système économique de l'époque, s'agissant particulièrement du régime des exportations de matières premières par les pays en développement, notamment en ces termes : «les pays capitalistes qui détiennent le pouvoir de décision, sont dans l'incapacité de maitriser les phénomènes économiques internationaux (inflation, fluctuations brusques et imprévues des prix des matières premières, pénurie alimentaire,(...). Les Etats du tiersmonde considèrent que le jeu incontrôlé de la loi du marché ne peut permettre de promouvoir le développement à l'échelle mondiale $\rangle^{17}$. Ils reprochaient à ce système économique international de maintenir et d'accroitre les inégalités et injustices dans la mesure où il ser-

14 L'analyse évaluative ou prescriptive a pout objet d'évaluer la règle juridique ou le concept juridique au regard de théories de la justice. Cette démarche consiste à constater la nature juste ou injuste de telle ou telle autre règle de droit international en se référant à une définition de la justice. Ainsi, le paradigme de responsabilité sociale d'entreprise constitue, pour nous, le substrat théorique de la justice. Dès lors, l'exercice critique consite à dénicher si les normes de responsabilité sociale répondent aussi au paradigme de marché gagnant-gagnant, du fait que le win-win est de nos jours considéré en relations internationales comme le modèle de marché juste qui permet à l'Afrique de tirer largement profit de ses ressources. L'analyse évaluative revêt tout de même une dimension empirique qui permet de tester ses postulats au regard du droit international positif, mais dans un regard prescriptif. Elle n'exclut pas en revanche l'approche descriptive qui joue un rôle subsidiaire dans le cadre de cette étude. Pour plus d'éclairage sur ce type d'analyse, lire CORTEN (O.), Méthodologie du droit international public, Editions de l'Université de Bruxelles, 2009, pp. 26-27.

15 Ces recommandations s'adressent aussi bien aux entreprises nationales que transnationales ou multinationales.

16 Sigle signifiant Responsabilité sociale des entreprises.

17 TOUSCOZ J., « La coopération internationale et les matières premières exportées par les pays du Tiers Monde : les conditions d'un échange égal », op.cit. 
vait les intérêts des pays capitalistes ${ }^{18}$.Ces préoccupations ont été officiellement exprimées à l'occasion de multiples conférences internationales réunies à l'initiative des pays du Tiers Monde ${ }^{19}$. Elles étaient réaffirmées avec force et intransigeance par le Président du Conseil révolutionnaire et du Conseil des Ministres d'Algérie, M. Houari Boumédienne, dans sa lettre au Secrétaire général des Nations Unies, demandant la convocation d'une session spéciale de l'Assemblée générale et dans son discours devant l'Assemblée au cours de la même session ${ }^{20}$. C'est sur ces bases qu'ont été élaborés la Déclaration et le Programme d'action, adoptés sans vote ${ }^{21}$. Aussi, les résultats de la session spéciale ont-ils été diversement interprétés : si pour les uns, cette session, marquée par l'irréalisme, a conduit à un échec en raison de l'absence de consensus $^{22}$; pour d'autres, au contraire, elle a consacré le poids du Tiers Monde et elle n'a constitué que le commencement d'un processus révolutionnaire.

En 1982, la Conférence des Nations Unies sur le commerce et le développement dénombrait $7000 \mathrm{STN}^{23}$. En 2010, elles sont 82000 contrôlant 810000 filiales, représentant près de $10 \%$ du PIB mondial et $33 \%$ du commerce international et sont essentiellement occidentales. Ce sont des « entités économiques ou un ensemble d'entités économiques opérant dans deux ou plusieurs pays, quel qu'en soit le cadre juridique, le pays d'origine ou d'établissement, que leur action soit prise individuellement ou collectivement. Les STN sont des personnes juridiques de droit privé avec une implantation territoriale multiple mais un centre unique pour les décisions stratégiques $»^{24}$. Elles peuvent fonctionner avec une société mère et des filiales, constituer des groupes de sociétés au sein d'un même secteur d'activité, des conglomérats ou coalitions ayant des activités diverses, s'unifier par le biais de fusions ou d'absorptions ou constituer des ensembles financiers (holdings). Ces sociétés peuvent élire domicile dans un ou plusieurs pays: dans celui du siège réel de l'entité mère, dans celui du siège des principales activités et/ou dans le pays où la société a été enregis-

18 L'écart des niveaux de vie se creuse, le transfert des richesses des pays en voie de développement vers les pays développés se poursuit, les pays en voie de développement qui comptent $70 \%$ de la population mondiale assurent moins de $7 \%$ de la population manufacturière totale et ce pourcentage ne s'est guère accru depuis vingt ans).

19 Les textes de base sont la Charte d'Alger, adoptée par la Conférence des Non-Alignés eя I973, la Charte des Droits et Devoirs économiques des Etats, adoptée par l'Assemblée générale le 12 décembre 1974, les projets de déclaration et de plan d'action concernant le développement et la coopération industrielle, établis par les 77 en janvier 1975 et la Déclaration et le Programme d'action.

20 Voir Chronique mensuelle de l'ONU, mai 1974, vol. XI, nº j, p. 34 et s.

2138 orateurs ont fait connaître leur position parmi lesquels les représentants des Etats-Unis, de la France, de la Grande- Bretagne, de la République fédérale allemande, de la Belgique, du Canada, de l'Australie, de la Suède et de la Communauté économique européenne notamment, et ont exprimé des réserves plus ou moins étendues et formelles.

22 L'irréalisme a dominé les débats de la session spéciale. Voir Marchés tropicaux, 10 mai 1974, n 1487, p. 1233.

23 Sigle signifiant Société transnationale.

24 CETIM, "Les sociétés transnationales, acteurs majeurs dans les violations des Droits de l’Homme », Cahier critique n¹0, décembre 2011, p. 35 
trée ${ }^{25}$. Ce sont des agents économiques soumis, en principe au droit d'un État et à la juridiction de ses tribunaux. Actuellement, les STN sont civilement, voire pénalement, responsables de violations des droits humains, au même titre que les personnes physiques, dans les États. Mais la société transnationale, n'a pas une personnalité particulière distincte de chacune de ses entités, si bien que ses dernières peuvent répondre de leurs actes de façon dispersée et tirer profits des intérêts opposés des États dans lesquels elles opèrent. La complexité structurelle et organique qui caractérise les STN est en sourdine d'une activité économique vorace au détriment des intérêts inaliénables des peuples.

C'est dans le but de minimiser l'impact négatif de l'activité des STN que le Conseil économique et social des Nations Unies (ECOSOC) créait en 1974 la Commission des sociétés transnationales. Elle état composée de 48 États, ayant pour missions prioritaires d'enquêter sur les activités des STN, d'élaborer un Code de conduite lequel était débattu durant dix ans sans aboutir. Le Centre des sociétés transnationales, organisme autonome au sein du Secrétariat de l'ONU, créé la même année, fonctionnait comme secrétariat de la Commission des sociétés transnationales. Entre 1993 et 1995, les deux organismes furent pratiquement démantelés et leurs objectifs changés. Le Centre sur les sociétés transnationales est devenu la Division des sociétés transnationales et de l'investissement au sein de la Conférence des Nations Unies sur le commerce et le développement (CNUCED).

De même, l'Assemblée générale de l'ONU avait également décidé de la transformation de la Commission des sociétés transnationales en Commission de l'investissement international et des sociétés transnationales en prenant en compte le « changement d'orientation » de la Commission. En 1997, lors de la préparation de l'Assemblée Générale des Nations Unies consacrée à l'évaluation des engagements cinq ans après le Sommet de Rio, le rôle des entreprises dans le développement durable est consacré et inscrit au programme de la sixième session de la Commission du développement durable en 1998. En 1998, il fut à nouveau question au sein des Nations Unies de mettre en place des normes internationales pour la régulation des activités des STN lorsque la Sous-commission de la lutte contre les mesures discriminatoires et de la protection des minorités adopta une résolution visant à étudier l'activité et les méthodes de travail des STN en rapport avec le plein exercice des droits économiques, sociaux et culturels ainsi que du droit au développement. La même année, la Global Reporting Initiative (GRI) est lancée par l'association américaine Coalition for Enviromentally Responsible Economies (CERES) sous l'égide du PNUE (Programme des Nations Unies pour l'Environnement), sur les rapports de développement durable et les systèmes de reddition (reporting). Aujourd'hui organisation indépendante, elle a pour mission d'élaborer et de mettre à jour en permanence, de diffuser des lignes directrices et des indicateurs mondialement utilisables pour aider les entreprises à produire des rapports de développement durable. 


\section{Enième échec d'une tentative d'institution des normes internationales contraignantes en matière de RSE}

En 2003, les Normes de l'ONU sur la responsabilité en matière de droits de l'homme des sociétés transnationales et autres entreprises sont adoptées à l'unanimité par la sous-commission de la promotion et de la protection des droits de l'homme, rendant les entreprises directement responsables de leurs agissements concernant les droits de l'homme, exigeant la transparence de leurs activités et des compensations en cas de violations des droits de l'homme. En juillet 2005, ce cadre, considéré comme trop contraignant par les représentants des sociétés y travaillant, la réflexion est relancée ; et le Secrétaire général des Nations Unies, M. Kofi Annan, nomme John Ruggie, en tant que Rapporteur spécial du Secrétaire général sur les droits de l'homme et les sociétés transnationales. Dans son rapport de 2008, J. Ruggie laisse entendre que les STN ne sont pas directement soumises au droit international et que la solution la plus appropriée serait que les États, avec les entreprises et la société civile, s'inspirent de quelques instruments internationaux pour établir des normes et initiatives non contraignantes juridiquement. De ces tractations naquit le Pacte Mondial (Global Compact), un code de conduite volontaire qui n'est ni juridiquement contraignant, ni un moyen de surveiller et de contrôler les entreprises, ni un système de gestion ni un organe de règlementation. Il représente cependant une avancée dans la légitimation des interventions des États dans la gouvernance des STN présentes sur leurs territoires.

Cette évolution s'inscrit dans le cadre du développement des notions de Responsabilité Sociale des Entreprises (RSE) et des Principes de transparence. Transposé à l'entreprise, le développement durable se traduit par la notion de Triple bottom line (Elkington, 1999) ${ }^{26}$ ou triple résultat, c'est-à-dire que l'entreprise socialement responsable doit être performante dans les trois dimensions que sont l'économique, le social/sociétal et l'environnemental, ce qui conduit à évaluer sa performance sous trois angles : la rentabilité économique, le respect de l'environnement, l'équité sociale.

Autrement dit, s'engager dans le développement durable consiste pour un dirigeant à veiller à la rentabilité économique de son activité tout en cherchant à minimiser son impact sur l'environnement et en prenant en compte les intérêts des parties prenantes. Ceux-ci apparaissent dans la seconde dimension qui englobe les conséquences sociales de l'activité de l'entreprise pour l'ensemble de ses parties prenantes : employés (conditions de travail, niveau de rémunération, non-discrimination, exclusion, chômage,...), fournisseurs, clients (sécurité et impacts psychologiques des produits), communautés locales (nuisances, respect des cultures) et la société en général.

En plus, l'entreprise est évaluée à partir de sa politique sociale et du respect des droits de l'Homme. L'intersection entre l'économique et le social concerne l'équité et la justice sociale et fait référence au respect des droits sociaux, au respect des règles de la diversité et de l'égalité des chances, à la valorisation du capital humain, à la participation aux résultats,

26 MARECHAL, «La dimension sociale du développement durable », Alternatives Economiques, $\mathrm{n}$ ${ }^{\circ} 191$, avril 2001, p. 15. 
etc... Le rapport entre le social et l'environnement renvoie aux conditions permettant de rendre viable l'activité humaine : sécurité, santé, gestion des risques professionnels et environnementaux, intégration de l'entreprise dans son bassin d'emploi, participation à la vie locale, etc... ${ }^{27}$ Plusieurs institutions internationales et cadres politiques internationaux ont suivi cette ligne, en mettant en place des principes directeurs, " recommandations que les gouvernements adressent aux entreprises multinationales où ils énoncent des principes et des normes volontaires de comportement des entreprises dans le respect des lois applicables » à l'endroit des STN. Il s'agit notamment de :

- l'Organisation de Coopération et de Développement Economique (OCDE), qui avec les Points de Contact Nationaux, évalue le respect de ses principes directeurs via des mécanismes d'enquête, de sanction et de réparation des abus, même si son pouvoir de sanction est limité;

- l'OIT, qui a fait une « Déclaration de principes tripartites sur les entreprises transnationales et la politique sociale », invitant au respect des principes directeurs, mais qui ne dispose pas de pouvoir de sanction

- l'ONU, qui édicte les principes à observer par les entreprises en matière des droits de l'Homme

Il existe d'autres normes de portée plus générale s'agissant de la protection des droits de l'homme, qu'il ne sera pas opportun d'examine ici, tel que les pactes internationaux relatifs aux droits sociaux-économiques, civiques et politiques. Mais alors quid de normes internationales relatives à la protection des droits de l'homme face aux industries des matières premières tenues

I. Les normes internationales relatives à la responsabilité sociale d'entreprise : reconnaissance des valeurs défendues par les pionniers de la Charte d'Alger

Il sied de signaler d'entrée de jeu que la revue du droit international positif ne renseigne pas sur l'existence des traités internationaux de portée multilatérale relatifs à l'industrie des matières premières. En revanche, l'on peut noter des normes non contraignantes en termes de directives, recommandations et des entités intergouvernementales instituées à cette fin, ayant trait essentiellement aux droits de l'homme dans leur dimension intégrale. Nonobstant la portée facultative de ces normes, les entreprises responsables des violations des droits de l'homme peuvent être condamnées conformément au droit international des droits de l'homme, à travers les avis du comité des droits de l'homme qui ne fait pas l'objet de la présente étude. Passons en revue quelques-unes d'elles.

27 ERNULT, J. et ASHTA, A., « Développement durable, responsabilité sociétale de l'entreprise, théorie des parties prenantes : Évolution et perspectives ", Cahiers du CEREN 21, 2007, pp. 4-31, spécialement p. 10. 


\section{Le Pacte mondial des Nations Unies}

Des défis sociaux, politiques et économiques complexes affectent les entreprises à travers le monde : pratiques liées à l'exploitation humaine, à la corruption, aux inégalités de revenus et obstacles divers décourageant l'innovation et l'esprit d'entreprise. Ainsi, beaucoup d'entreprises reconnaissent la nécessité de collaborer avec les gouvernements, la société civile, les travailleurs, les syndicats et les Nations Unies afin de contribuer à la construction des sociétés prospères et durables ${ }^{28}$. D'où le Pacte mondial ou Global Compact qui se veut une initiative internationale d'engagement volontaire en matière de responsabilité sociétale de l'entreprise (RSE).

Créé à l'initiative des Nations Unies en 2000, le Pacte Mondial est, « un pacte par lequel des entreprises s'engagent à aligner leurs opérations et leurs stratégies sur dix principes universellement acceptés concernant les droits de l'homme, les normes du travail, l'environnement et la lutte contre la corruption $"{ }^{29}$. L'objectif est double, inciter les entreprises dans le monde entier à conduire leurs activités en respectant les Dix Principes énoncés et mobiliser l'action à l'appui des grands objectifs des Nations Unies, y compris les objectifs du Millénaire pour le développement. Le Pacte Mondial a notamment pour avantage d'accueillir des participants autres que les entreprises, permettant ainsi aux acteurs de la société civile de "donner des conseils d'expert, demander des comptes aux entreprises quant à leurs engagements et à leurs manquements, encourager les initiatives responsables, apporter des connaissances qui peuvent faciliter l'application du Pacte ».

L'idée du Pacte mondial des Nations Unies avait été lancée en 1999, par le Secrétaire général de l'ONU, Kofi Annan, lors du Forum économique de Davos et la phase opérationnelle a débuté en juillet 2000 à New York. C'est un réseau regroupant le Bureau du Pacte mondial, les entreprises volontaires et quatre organismes des Nations Unies ${ }^{30}$. Le Pacte mondial n'est pas un instrument de régulation, plutôt un cadre facultatif ouvert aux entreprises essentiellement multinationales. Le Pacte invite les entreprises à adopter, soutenir et mettre en oeuvre, dans leur sphère d'influence, un ensemble de valeurs fondamentales en matière de droits de l'homme, de normes du travail, d'environnement et de lutte contre la corruption.

En matière des Droits de l'homme, deux principes sont édictés : le premier invite les entreprises à promouvoir et à respecter la protection du droit international relatif aux droits de l'homme; et le second les encourage à veiller à ne pas se rendre complices de violations

28 Voir le site de Pacte Mondial Réseau France, http://www.pactemondial.org/, consulté le 10 janvier 2015.

29 Bureau du Pacte mondial des Nations Unies, L'entreprise citoyenne dans l'économie mondiale. Le Pacte Mondial des Nations Unies, disponible en ligne sur http://www.unglobalcompact.org/langua ges/french/index.html. consulté le 13 décembre 2014.

30 Le Haut Commissariat des Nations Unies aux droits de l'homme, le Programme des Nations Unies pour l'environnement, l'Organisation internationale du travail (OIT) et le Programme des Nations Unies pour le développement. 
des droits de l'homme. Quatre principes sont énoncés dans le domaine de Droit du travail : les entreprises sont invitées à respecter la liberté d'association et à reconnaître le droit de négociation collective; éliminer toutes les formes de travail forcé ou obligatoire; abolir effectivement le travail des enfants; éliminer toute discrimination en matière d'emploi et de profession.

Dans le pilier de l'environnement, les entreprises sont invitées à adopter le principe de précaution face aux problèmes d'environnement; à prendre des initiatives tendant à promouvoir une plus grande responsabilité en matière environnementale; et à favoriser la mise au point et la diffusion de technologies respectueuses de l'environnement. Dans le domaine de lutte contre la corruption, les entreprises sont invitées à lutter contre la corruption sous toutes ses formes, y compris l'extorsion de fonds.

Le Pacte est une initiative volontaire des entreprises qui s'engagent à prendre des mesures pour modifier leur fonctionnement, de façon à intégrer ces principes à leur stratégie et à leurs activités quotidiennes. Elles doivent décrire dans un rapport annuel comment elles mettent en oeuvre les principes du Pacte mondial, sous la forme d'un engagement général appelé "Communication sur le progrès », et rédiger chaque année un exemple de bonne pratique rendant compte de progrès sur au moins un des principes. Des indicateurs de performance sont prévus pour chaque principe afin de permettre aux entreprises de s'auto-évaluer. Il s'agit de documents qui contiennent des règles relatives au travail, aux conditions des travailleurs. Ces documents se caractérisent par une grande diversité matérielle. Sur le fond, ils comportent des engagements à respecter un principe, une politique, une action, ou le bilan des réalisations concrètes. Le plus souvent, les deux discours, engagements-réalisations, sont mêlés. Ce qui permet de distinguer les documents, c'est le caractère plus ou moins concret de leur contenu. On trouve ainsi de pures déclarations d'engagements, vagues et générales, des lettres d'engagement du dirigeant de l'entreprise, d'adhésion ou de renouvellement d'adhésion aux principes du pacte. ${ }^{31} \mathrm{Il}$ importe de préciser que ces documents sont donnés par les entreprises comme des exemples de bonnes pratiques et ne donnent pas une idée exhaustive de la politique suivie par ces entreprises en matière de RSE. Leur choix est soumis à la subjectivité de l'entreprise, ce qui correspond à l'esprit du Pacte qui n'impose aucune directive en la matière. Une seule « bonne pratique » peut suffire à satisfaire les engagements d'une entreprise. L'on se rend bien compte de la nécessité des normes coercitives si la responsabilité sociale d'entreprise ne veut demeurer utopique. A coté du Pacte, se juxtaposent d'autres normes qu'il convient de passer en revue.

2. Les Lignes directrices de l'OCDE pour les entreprises multinationales

Communément appelés Principes directeurs de l'OCDE, il s'agit de recommandations que les gouvernements adressent aux entreprises multinationales exerçant leurs activités dans

31 MAZUYER, J., L'application du Pacte mondial des Nations Unies par les entreprises françaises : un exemple de " bonnes pratiques » de RSE?, 2012, p. 2. 
les pays adhérents ou à partir de ces derniers. Ils contiennent des principes et des normes non contraignants destinés à favoriser une conduite raisonnable des entreprises dans un environnement mondialisé, en conformité avec les législations applicables et les normes internationalement admises». ${ }^{32}$

Les Principes directeurs constituent, en matière de conduite responsable des entreprises, le seul code exhaustif convenu à l'échelon multilatéral que les gouvernements se sont engagés à promouvoir. Les recommandations énoncées dans les Principes directeurs expriment les valeurs partagées par les gouvernements des pays dont provient une grande partie de l'investissement direct international et dans lesquels sont implantées nombre d'entreprises multinationales les plus importantes. Ces Principes ont vocation à promouvoir une contribution positive des entreprises au progrès économique, environnemental et social partout dans le monde.

Cependant, il ya lieu de relever de prime à bord que les Principes directeurs sont des simples recommandations que les gouvernements adressent conjointement aux entreprises multinationales. Ils énoncent des principes et des normes de bonnes pratiques conformes aux législations en vigueur et aux autres normes internationalement admises. Le respect des Principes directeurs par les entreprises est volontariste et n'est pas juridiquement contraignant. Néanmoins, certains sujets abordés dans les Principes directeurs de l'OCDE peuvent également être réglementés par des législations nationales ou des engagements internationaux dont la violation engage la responsabilité de l'Etat ou de l'entreprise. Ensuite, les entreprises ont $l^{\prime}$ 'obligation de se conformer à la législation de leur pays.

Les Principes directeurs de l'OCDE ne sauraient se substituer à une législation ou une réglementation nationale, ni prévaloir sur elle. Si les Principes directeurs vont au-delà de la loi dans de nombreux cas, ils ne devraient pas, et tel n'est pas leur but, placer les entreprises dans une situation où elles feraient face à des obligations contradictoires. Les Principes directeurs ne visent pas à instaurer des différences de traitement entre les entreprises multinationales et les entreprises nationales; ils traduisent des pratiques pouvant être recommandées à toutes. L'on attend donc des entreprises multinationales et nationales qu'elles aient le même comportement dans tous les cas où les Principes directeurs s'appliquent aux unes et aux autres.

32 OCDE, Les principes directeurs de l'OCDE à l'intention des entreprises multinationales, Editions OCDE, 2011, disponible en ligne sur http://www.oecd.org/fr/daf/inv/mne/48004355.pdf, consulté le 20 novembre 2015. 
3. Les Principes directeurs des Nations Unies relatifs aux entreprises et aux droits de l'Homme

Les principes directeurs des Nations Unies relatifs aux entreprises et aux droits de l'Homme résultent du rapport John Ruggie, alors Représentant spécial du Secrétaire général chargé de la question des droits de l'Homme et des sociétés transnationales et autres entreprise ${ }^{33}$.

Les Principes directeurs relatifs aux entreprises et aux droits de l'homme des Nations unies s'inspirent de la Charte des Nations unies relative aux droits de l'homme et de la Déclaration de l'OIT (Organisation internationale du travail) relative aux principes et droits fondamentaux au travail. Ils s'appliquent à tous les États et à toutes les entreprises, indépendamment de leur taille, de leur secteur, de leur milieu d'implantation, de leur régime de propriété ou de leur structure, et quelle que soit la capacité et/ou la volonté des États à honorer leurs propres obligations en matière de respect des droits de l'homme ${ }^{34}$.

Le 17 juin 2011, le Conseil des droits de l'Homme des Nations Unies a adopté des « Principes directeurs sur les droits de l'Homme et les entreprises ». Cette adoption à l'unanimité est, à juste titre, considérée comme un évènement majeur pour la protection des droits de l'Homme et pour l'évolution du concept de responsabilité sociale des entreprises. Il a fallu 18 ans pour arriver à ce résultat; l'idée avait été lancée par la conférence mondiale des droits de l'Homme de Vienne, dès 1993, qu'il fallait entraîner ces acteurs majeurs de l'économie et du social que sont les entreprises à s'engager au service du respect des droits de l'Homme. Plusieurs tentatives se sont succédées, confiées à des rapporteurs et comités ad hoc. En 1999, le Secrétaire Général des Nations Unies a lancé le Pacte Mondial, dont 4 des 10 Principes sont relatifs aux droits de l'Homme. Puis la Sous-commission des droits de l'Homme des Nations Unies a tenté d'élaborer un traité sur le sujet, que les États ont, à travers la Commission des droits de l'Homme, refusé d'examiner en 2004. Le Conseil des droits de l'Homme, nouvelle appellation de la Commission, mandate en juin 2005 un « Représentant spécial du secrétaire général des Nations Unies pour les droits de l'Homme, les entreprises transnationales et autres entreprises », le professeur John Ruggie, universitaire connu pour avoir participé à l'élaboration du Pacte Mondial. Au terme d'un premier mandat de trois ans, John Ruggie a fait adopter en juin 2008 par le Conseil un « cadre conceptuel et des principes d'action afin d'ancrer le débat sur les entreprises et les droits de l'homme ». Il s'articule autour de trois principes fondamentaux :

1. l'obligation de protéger incombant à l'État lorsque des tiers, y compris des sociétés, portent atteinte aux droits de l'homme;

33 Le Haut Commissariat des Nations Unies aux droits de l'homme, Principes directeurs relatifs aux entreprises et aux droits de l'homme: mise en cuvre du cadre de référence " protéger, respecter et réparer» des Nations Unies, New York et Genève, 2011.

34 OXFAM, «Oxfam International's Perspective on the Draft Guiding Principles for the mplementation of the United Nations "Protect, Respect and Remedy" Framework », 2011, disponible en ligne sur http://www.oxfamamerica.org/files/oxfam-ruggie-guiding-principles.pdf, consulté le 10 février 2015. 
2. la responsabilité des entreprises de respecter les droits de l'homme;

3. la nécessité d'un accès plus effectif à des mesures de réparation pour les victimes.

Suite à de très nombreuses concertations avec des institutions internationales engagées dans la construction d'autres normes (ISO, OCDE, Banque Mondial, Union Européenne), un consensus associant organisations d'employeurs, d'employés et de défense des droits de l'Homme s'est construit, débouchant sur l'adoption unanime par le Conseil des droits de l'Homme, en juin 2011, des Principes directeurs sur les entreprises et les droits de l'Homme. Ils sont organisés en trois piliers déduits du cadre de 2008 : «protéger, respecter, remédier $\gg$.

Les principaux apports de ces Principes directeurs sont :

1. L'affirmation du rôle central de l'Etat dans la protection et la promotion des droits de l'Homme vis-à-vis des entreprises;

2. La priorité donnée à l'approche par les risques;

3. la responsabilité étendue à la chaîne de valeur;

4. le droit international des droits de l'Homme et du droit du travail pris comme référence. Ce document énonce d'abord les obligations de protéger les droits de l'Homme incombant à l'Etat, fixe ensuite la responsabilité incombant aux entreprises de respecter les droits de l'homme et donne des orientations relatives aux voies de recours ouvertes aux parties.

S'agissant de voies de recours, les Etats doivent, au titre de leur obligation de protéger contre les atteintes aux droits de l'Homme commises par des entreprises, prendre des mesures appropriées pour assurer, par le biais de moyens judiciaires, administratifs, législatifs ou autres, que lorsque de telles atteintes se produisent sur leur territoire et/ou sous leur juridiction, les parties touchées ont accès à un recours effectif. Tel est le principe fondateur. Il devrait être institué des mécanismes judiciaires et non judiciaires relevant de l'Etat. Les États devraient prendre des mesures appropriées pour assurer l'efficacité des mécanismes judiciaires internes lorsqu'ils font face à des atteintes aux droits de l'Homme commises par des entreprises, y compris en examinant les moyens de réduire les obstacles juridiques, pratiques et autres qui pourraient amener à refuser l'accès aux voies de recours.

De ce point de vue, il parait judicieux d'établir des mécanismes judiciaires effectifs pour assurer l'accès aux voies de recours. La capacité de ces mécanismes à remédier aux atteintes aux droits de l'Homme commises par des entreprises dépend de leur impartialité, de leur intégrité et de leur capacité à garantir une procédure régulière. Les États devraient veiller à ne pas ériger d'obstacles propres à empêcher que des recours soient formés devant les tribunaux pour des affaires légitimes lorsque le recours judiciaire est un élément essentiel de l'accès à des mesures de réparation ou lorsqu'il n'y a pas d'autres possibilités de recours effectif. Ils devraient aussi veiller à ce que le cours de la justice ne soit pas entravé par la corruption de la procédure judiciaire, à ce que les tribunaux soient à l'abri des pressions économiques et politiques d'autres agents de l'État et acteurs économiques et à ce 
qu'il ne soit pas fait obstacle aux activités légitimes et pacifiques des défenseurs des droits de l'Homme. ${ }^{35}$

Des obstacles juridiques pouvant empêcher l'instruction d'affaires d'atteintes aux droits de l'homme commises par les entreprises apparaissent lorsque, par exemple: la manière dont la responsabilité juridique est répartie entre les membres d'un groupe d'entreprises en vertu du droit pénal et civil interne permet d'éviter plus facilement d'établir correctement les responsabilités; les requérants s'exposent au déni de justice dans un État d'accueil et ne peuvent pas accéder aux tribunaux des États d'origine quel que soit le bien-fondé de la plainte; certains groupes, comme les peuples autochtones et les migrants, ne peuvent pas profiter du niveau de protection juridique des droits de l'Homme dont bénéficie la population dans son ensemble.

Des obstacles d'ordre pratique et procédural à l'accès au recours judiciaire peuvent apparaître lorsque, par exemple: les coûts afférents au dépôt d'une plainte ne dissuadent pas seulement les requérants de porter plainte pour des affaires dénuées de fondement et/ou ne peuvent pas être réduits à des niveaux raisonnables par le biais d'un soutien du gouvernement, de mécanismes fondés sur le marché (comme l'assurance contre les litiges et les barèmes de frais de justice) ou d'autres moyens; les requérants ont des difficultés à obtenir une représentation juridique par suite d'un manque de ressources ou d'autres facteurs incitant les juristes à les conseiller dans ce domaine; les possibilités qui existent de regrouper les plaintes ou de permettre les procédures de groupe (comme les actions catégorielles et autres procédures en nom collectif) sont insuffisantes, empêchant ainsi les requérants à titre individuel d'accéder à des voies de recours efficaces; les procureurs de la République n'ont pas les ressources, les compétences et le soutien nécessaires pour honorer les obligations propres de l'État d'enquêter sur la participation des individus et des entreprises aux délits liés aux droits de l'Homme ${ }^{36}$.

Bon nombre de ces obstacles résultent des déséquilibres fréquents observés du point de vue des ressources financières, de l'accès à l'information et à des conseils d'experts entre les parties qui déposent des plaintes pour atteintes aux droits de l'homme commises par des entreprises, ou viennent s'ajouter à ces disparités. A cause d'une discrimination active, ou de conséquences fortuites des modes de conception et de fonctionnement des mécanismes judiciaires, des individus issus de groupes ou de populations plus exposés à la vulnérabilité et à la marginalisation font souvent face à des obstacles culturels, sociaux, physiques et financiers supplémentaires pour accéder à ces mécanismes, les utiliser et en bénéficier. Une attention particulière devrait être accordée aux droits et aux besoins spécifiques de ces groupes ou populations à chaque étape du processus de réparation: accès, procédures et issue du recours. ${ }^{37}$

35 Ibidem, p. 33.

36 Ibidem.

37 Ibidem, p. 34. 
Il devrait également exister des mécanismes de réclamation qui ne relèvent pas de l'Etat et cette catégorie des mécanismes comprend les mécanismes administrés par une entreprise à titre individuel ou avec des parties prenantes, par une association professionnelle ou un groupe multipartite. Ils sont non judiciaires, mais peuvent faire appel à des procédures judiciaires, fondés sur le dialogue ou d'autres processus culturellement adaptés et compatibles avec le Droit. Ces mécanismes peuvent offrir des avantages particuliers comme la rapidité d'accès et de réparation, des coûts réduits et/ou une portée transnationale. ${ }^{38}$

Une autre catégorie comprend les organes régionaux et internationaux de défense des droits de l'Homme. Ceux-ci ont le plus souvent traité des violations présumées des obligations qui incombent aux États de respecter les droits de l'Homme. Toutefois, certains ont aussi traité des cas dans lesquels des États ne se sont pas acquittés de l'obligation de protéger contre les atteintes aux droits de l'Homme commises par des entreprises. Les États peuvent jouer un rôle utile en faisant mieux connaître ces options, ou en en facilitant l'accès, en complément des mécanismes qu'ils fournissent ${ }^{39}$.

Pour pouvoir examiner rapidement les plaintes et y remédier directement, les entreprises devraient établir des mécanismes de réclamation au niveau opérationnel ou y participer pour les individus et les collectivités qui risquent d'être lésés. Les mécanismes de réclamation de niveau opérationnel sont directement accessibles pour les individus et les collectivités qui risquent de pâtir de l'activité d'une entreprise. Ils sont généralement administrés par des entreprises, seules ou en collaboration avec d'autres, y compris avec les acteurs concernés. Ils peuvent aussi être assurés en recourant à un expert ou un organe extérieur mutuellement acceptable. Ils n'exigent pas que les plaignants se dirigent d'abord vers d'autres voies de recours. Ils peuvent demander directement aux entreprises d'examiner les problèmes et de réparer les préjudices subis ${ }^{40}$. Il convient à présent de voir un autre instrument de grande valeur en matière de RSE

4. La déclaration tripartite de l'OIT sur les entreprises multinationales et la politique sociale

Face à la montée en puissance des multinationales dans les années soixante-dix, des défis nouveaux ont surgi et donné lieu à des discussions soutenues qui ont conduit à l'élaboration d'instruments internationaux visant à fixer à leur intention des règles de conduite et à définir les modalités de leurs relations avec les pays hôtes, principalement dans le monde en développement. Les problèmes posés par les activités de ces entreprises touchaient au travail et à la politique sociale. L'organisation internationale du travail, OIT, a initié des travaux dont l'objectif était « l'établissement de directives internationales dans son domaine

39 Ibidem.

40 Ibidem. 
de compétence $»^{41}$. Cette initiative aboutit à l'adoption, en 1977, par le Conseil d'administration du Bureau international du Travail, de la Déclaration de principes tripartite sur les entreprises multinationales et la politique sociale (la Déclaration sur les EMN). Les principes énoncés par cet instrument de portée universelle sont destinés selon les dires mêmes de l'organisation « à guider les entreprises multinationales, les gouvernements, les employeurs et les travailleurs dans des domaines tels que l'emploi, la formation, les conditions de travail et de vie ainsi que les relations professionnelles $\gg{ }^{42} \mathrm{La}$ déclaration a été révisée en 2000 pour inclure les principes fondamentaux et droits liés au travail, puis en 2006 pour une mise à jour des instruments de l'OIT auxquels elle fait référence.

41 OIT, Déclaration de principes tripartite sur les entreprises multinationales et la politique sociale, adoptée par le Conseil d'administration du Bureau international du Travail à sa $204^{\mathrm{e}}$ session (Genève, novembre 1977), telle qu'amendée par le Conseil à sa $279^{\mathrm{e}}$ session (Genève, novembre 2000) et à sa $295^{\mathrm{e}}$ session du 28 mars 2006, disponible en ligne http://www.ilo.org/wcmsp5/groups /public/_ed_emp/—mp_ent/documents/publication/wcms_124923.pdf, consulté le 28 décembre 2014.

42 Idem. 
Selon Isabelle Daugareilh, «cet instrument conçoit la RSE dans quatre domaines : l'emploi ${ }^{43}$, la formation ${ }^{44}$, les conditions de travail ${ }^{45}$ et de vie ainsi que les relations professionnelles $^{46}$ ». Ses dispositions s'appuient sur celles d'une série de conventions et de recommandations internationales du travail que les partenaires sociaux sont constamment priés d'avoir à l'esprit et d'appliquer dans toute la mesure du possible ${ }^{47}$. L'adoption en 1998 de la Déclaration de l'OIT relative aux principes et droits fondamentaux au travail a souligné combien le respect des conventions fondamentales est important pour la réalisation des objectifs de l'OIT, et c'est pourquoi la Déclaration sur les EMN tient compte des objectifs de la Déclaration de 1998.

Aujourd'hui, étant donné le rôle prédominant que jouent les entreprises multinationales dans le processus de mondialisation économique et sociale, l'application des principes de la

43 Promotion de l'emploi, égalité des chances et de traitement, sécurité de l'emploi... plus de détails, voir OIT, Déclaration de principes tripartite..., p. 4-5.

44 Les gouvernements devraient, en coopération avec toutes les parties intéressées, développer des politiques nationales de formation et d'orientation professionnelles étroitement liées à l'emploi (Convention $\mathrm{n}^{\circ} 142$ concernant le rôle de l'orientation et de la formation professionnelles dans la mise en valeur des ressources humaines, 1975, et recommandation $\left(n^{\circ} 195\right)$ sur la mise en valeur des ressources humaines: éducation et formation tout au long de la vie, 2004, rappelant la nature volontaire du contenu de la négociation collective et du niveau auquel elle est menée). Tel est le cadre dans lequel les entreprises multinationales devraient poursuivre leur politique de formation. Les entreprises multinationales devraient veiller, en déployant leurs activités, à ce que leurs travailleurs bénéficient à tous les niveaux, dans le pays d'accueil, d'une formation appropriée en vue de répondre aux besoins de l'entreprise ainsi qu'à la politique de développement du pays. Cette formation devrait, dans la mesure du possible, développer des aptitudes utiles en général et promouvoir les possibilités de carrière. Cette responsabilité devrait s'exercer, le cas échéant, en coopération avec les autorités du pays, les organisations d'employeurs et de travailleurs et les institutions locales, nationales ou internationales compétentes. Les entreprises multinationales exerçant leur activité dans des pays en développement devraient participer de même que les entreprises nationales à des programmes, y compris des fonds spéciaux, encouragés par les gouvernements des pays d'accueil et soutenus par les organisations d'employeurs et de travailleurs. Ces programmes devraient viser à encourager l'acquisition et le développement des compétences et à fournir une orientation professionnelle. Ils devraient être administrés en commun par les parties qui les soutiennent. Autant que praticable, les entreprises multinationales devraient, à titre de participation au développement national, fournir les services de personnel de formation qualifié pour aider à mettre en oeuvre les programmes de formation organisés par les gouvernements.

Les entreprises multinationales devraient, en coopération avec les gouvernements et dans la mesure admissible pour ne pas entraver le fonctionnement efficace de l'entreprise, offrir dans l'entreprise toute entière des possibilités pour élargir l'expérience des cadres de direction locaux dans des domaines appropriés tels que les relations professionnelles.

45 Il s'agit des aspects liés aux salaires, prestations, l'âge minimum, la sécurité et l'hygiène au travail.... p. 8-9.

46 Elles concernent la liberté syndicale, le droit d'organisation, la négociation collective, la consultation, l'examen des réclamations et les règlements des conflits du travail..., OIT, Déclaration de principes tripartite..., p. 10.

47 DAUGAREILH, I., Responsabilités de l'entreprise transnationale et globalisation de l'économie, Paris-Bruxelles, Bruylant-LGDJ, 2010, p. 25. 
Déclaration sur les EMN est aussi nécessaire et d'actualité que lors de l'adoption de cet instrument. A l'heure où s'intensifient dans de nombreuses régions du monde, les efforts faits pour attirer et encourager les investissements directs étrangers, une nouvelle occasion s'offre à toutes les parties de s'inspirer des principes de la Déclaration pour renforcer les effets positifs des activités des entreprises multinationales dans le domaine social et le domaine du travail.

Pour suivre l'effet donné à la Déclaration par les entreprises multinationales, les gouvernements et les organisations d'employeurs et de travailleurs, le BIT conduit une enquête périodique. Un résumé et une analyse des réponses reçues sont soumis au Conseil d'administration pour discussion. En cas de différend relatif à l'application de la Déclaration, les parties peuvent, grâce à une procédure instituée en 1981, adresser au BIT une demande d'interprétation de ses dispositions. Une assistance et des conseils peuvent être obtenus auprès du BIT en ce qui concerne la soumission des demandes d'interprétation. La Déclaration établit des principes de politique sociale dans un secteur d'activité sensible et fort complexe $^{48}$. L'adhésion à ses principes par toutes les parties concernées contribuerait à l'instauration d'un climat plus propice à la croissance économique et au progrès social. Mais, ce n'est qu'une déclaration!

La déclaration tripartite n'étant pas un instrument contraignant, il ne s'agit pas d'une réelle «procédure de résolution de litige pour non-respect d'une norme ${ }^{49}$. Néanmoins, elle permet de faciliter le dialogue entre employeurs et syndicats tout en donnant à ces derniers une arme supplémentaire dans leurs revendications. Si elle a le mérite de ne pas être trop longue (délai de traitement d'un an), ce mécanisme soufre d'un manque de popularité auprès des Etats et des syndicats comme le démontre le nombre peu élevé de saisine du bureau malgré l'ancienneté de l'instrument. Le fait de limiter les possibilités de requête aux situations concrètes est aussi à déplorer.

Un accord entre l'organisation et l'Etat peut permettre l'instauration d'un mécanisme de plainte au profit des personnes victimes de travail forcé. Cette procédure est ouverte aux victimes, mais aussi aux membres de leurs familles ainsi qu'à toute personne ayant un intérêt à agir. Le gouvernement peut s'engager à ce que les plaignants soient à l'abri de toute représaille ${ }^{50}$.

Il convient de signaler par ailleurs que l'Afrique, en tant que région, n'et pas restée en marge du mouvement international d'affirmation de la RSE et de développement industriel ; elle a, à cette fin, initié et institué des mécanismes.

49 Ibidem.

50 ILO, « Forced Labour Mechanism », disponible en ligne surhttp://www.ilo.org/yangon/complaints /lang-en/index.htm. Consulté le 19 novembre 2014. 


\section{Quelques politiques de développement industriel africain}

Les initiatives et politiques passées en matière de développement industriel ou ayant une composante de développement industriel de l'Afrique (DDIA, PAL et AIA), n'ont pas été individuellement et collectivement à la hauteur des attentes au regard des résultats obtenus après plus de vingt cinq ans.

Malgré les avantages comparatifs de l'Afrique de l'ouest dans bien des domaines, (production de matières premières, etc.), ni le plan d'action de Lagos (PAL, adopté en 1980), ni aucune des deux Décennies du développement industriel de l'Afrique (DDIA I, 1982-1992; et DDIA II, 1993-2003), n'a permis d'atteindre des résultats tangibles. Aucune de ces initiatives n'a réussi à former et maintenir sur place en Afrique, le capital humain critique de compétences et à mobiliser les ressources financières et de l'investissement en vue de doter l'Afrique des moyens techniques et d'infrastructures matérielles et institutionnelles nécessaires au soutien du développement industriel.

La Conférence Africaine des Ministres en charge de l'Industrie (CAMI) a procédé à l'évaluation de la DDIA I avant de lancer la DDIA II qui fut abandonnée à mi-parcours, faute de résultats satisfaisants. Ces différentes initiatives ont été conduites avec l'ONUDI, la CEA et le Secrétariat de l'OUA comme agences d'exécution, dont les contributions n'ont pas permis d'atteindre les résultats escomptés.

La conjonction de plusieurs facteurs favorables en 2000 (le renouvellement de la classe politique dirigeante dans les pays africains, etc.) a favorisé la création, à la fin de la DDIA II, de la Nouvelle Initiative Africaine (NAI) adoptée par les chefs d'Etat africains lors de leur sommet de Lusaka, transformée par ceux-ci, le 23 octobre 2001 à Lagos, en «Nouveau partenariat pour le développement africain » ou NEPAD.

\section{La Vision minière pour l'Afrique: l'émergence d'un paradigme répondant au contexte africain}

La Vision minière pour l'Afrique (VMA) a été adoptée par les chefs d'État et de gouvernement d'Afrique en février 2009. Cette Vision constitue la réponse des ministres africains au paradoxe de vivre dans un continent où se côtoient d'immenses richesses en ressources naturelles, une pauvreté envahissante et de grandes disparités. C'est une approche novatrice en ce qu'elle va au-delà de l'amélioration des régimes miniers nationaux pour déterminer comment l'exploitation minière peut contribuer véritablement au développement local, national et régional. À l'échelle locale, elle examine comment les travailleurs et les communautés locales peuvent tirer des bénéfices durables de l'exploitation minière tout en protégeant leur environnement. Au niveau national, elle étudie comment les pays peuvent mieux négocier les contrats avec les entreprises afin de générer des revenus équitables de l'extraction des 
ressources et soutenir l'approvisionnement local. Enfin, elle se penche sur l'intégration de l'exploitation minière aux politiques commerciales et industrielles régionales ${ }^{51}$.

La VMA propose un changement de paradigme qui abandonne le modèle d'exploitation des ressources extractives largement dépendant des marchés internationaux d'exportation. Ce modèle n'a jamais pu offrir à l'Afrique un développement socio-économique. La Vision aborde, dans une approche critique et prospective, le paradoxe qui oppose les ressources naturelles abondantes de l'Afrique et la pauvreté généralisée de ses populations. Ainsi que le précisait Carlos Lopez, sous-secrétaire des Nations-Unies et secrétaire exécutif à la CENUA « (...) le modèle de développement par l'exploitation des ressources qui est en place ne réussit pas à créer plus d'équité ni à enrichir le développement ${ }^{52}$.

Le but principal de la VMA est donc de délaisser le paradigme dominant de développement basé sur «l'exploitation des ressources» pour adopter un modèle qui favorise "une transformation structurelle des économies d'Afrique». Les ressources minérales serviraient de catalyseur à une croissance multisectorielle inclusive et au développement des marchés des ressources en Afrique, ce qui permettrait une diversification et une industrialisation économiques à l'échelle continentale. Par des réformes en matière de politiques, la VMA vise à corriger les défauts structurels du présent modèle, hérité de l'époque coloniale et qui se caractérise par « un secteur minier enclavé, des approches mono sectorielles et non intégrées à des objectifs plus vastes de développement, des capacités institutionnelles affaiblies et des relations de négociation profondément asymétriques entre gouvernements et compagnies $^{53}$. La VMA conçoit la RSE plutôt comme un outil pour renforcer la capacité des États de réaliser par eux-mêmes leurs priorités de développement et de s'acquitter de leurs responsabilités sociales et économiques. On s'attend à ce que les entreprises accomplissent leurs obligations en matière d'impôts de façon juste et transparente, et qu'elles paient des redevances équitables.

Dogbevi ${ }^{54}$ exprime des inquiétudes quant à la capacité des dirigeants africains à repousser les pressions externes exercées par certains donateurs qui appuient d'autres démarches d'extraction minière et de développement qui s'inscrivent à contre-courant de la VMA : les traités bilatéraux d'investissements que signent les pays d'Afrique pour favoriser les inves-

51 La VMA a été rédigée pour la première fois par l'équipe technique établi par l'Union africaine (UA) et la Commission Économique des Nations Unies pour l'Afrique (CENUA), avec l'aide de représentants du Partenariat minier africain (le forum intergouvernemental des ministres africains responsables du secteur minier), la Banque africaine de développement (BAD), la CNUCED et l'ONUDI. Plus de renseignements sur la VMA et le rapport du groupe international de recherche se trouvent au http://www.africaminingvision.org/.

52 DOGBEVI, E., «What Carlos Lopes said at the start of ADF VIII», ADF TODAY, 25 octobre, 2012.

53 CAMPBELL, B., Étude sur le rôle du secteur privé dans la réalisation des intérêts du Canada en développement international, Le Comité permanent des affaires étrangères et du développement international, 4 avril, 2012.

54 DOGBEVI, E., African Mining Vision not widely known Graham, ADF TODAY, 25 octobre, 2012, p. 30 . 
tissements étrangers, en illustrent la triste réalité. L'auteur s'interroge sur les répercussions de ces accords sur une politique industrielle susceptible de profiter aux peuples autochtones et, éventuellement sur le développement des entreprises locales et les possibilités de valorisation des minéraux. Comment les Accords de partenariat économique de 1'Union européenne affecteront-ils à l'avenir l'espace politique nécessaire à la réalisation de certains aspects de la VMA? De quelle façon tiendra-t-on compte de la VMA dans la création d'une zone de libre-échange en Afrique? Ce questionnement fera probablement l'objet d'une étude ultérieure.

\section{Le NEPAD et ses initiatives en faveur du développement industriel}

Tirant les leçons des initiatives devancières en matière de développement, le NEPAD exige au niveau de chaque région africaine une vision territoriale dont la mise en oeuvre en Afrique de l'Ouest est confiée à la CEDEAO. Ces efforts visent la consolidation d'une économie d'échelle et des pratiques de bonne gouvernance, une plus grande participation du secteur privé dans la réalisation des investissements intégrateurs en harmonie avec les interventions du secteur public.

C'est dans ce cadre que les ministres africains de l'Industrie ont décidé ensemble de lancer l'Initiative pour le renforcement des capacités productives en Afrique (IRCPA) qui a été adoptée par le Sommet des Chefs d'Etat et de Gouvernement de l'UA, en tant que partie du volet industriel du NEPAD. Il s'agit de :

- relancer le processus de changement structurel en Afrique;

- promouvoir le savoir faire économique et le développement durable;

- promouvoir les échanges intra régionaux et l'organisation de la production au niveau régional en mettant l'accent sur les secteurs de l'agro-alimentaire et du coton/textile ainsi que la mise en place d'une facilité financière.

Par ailleurs, l'Initiative pour l'Accès au Marché du NEPAD a notamment suscité beaucoup d'intérêt au sein de la communauté internationale de développement et des organisations, notamment l'ONUDI, qui ont lancé des initiatives dans le cadre du renforcement des capacités commerciales en vue de soutenir les pays en développement dans leurs efforts visant à avoir un meilleur accès aux marchés internationaux. Aussi, une étroite coopération est-elle nécessaire entre le NEPAD, les organisations régionales et internationales, comme l'ONUDI pour coordonner ces initiatives de renforcement des capacités de production et de développement du commerce. Ces initiatives permettront ainsi de relever de manière concertée le défi du développement des capacités de production en vue d'un meilleur accès aux marchés et d'une participation accrue au commerce international.

Dans le cadre d'une étude ultérieure, il sera démontré que les partenaires de l'Afrique ne sont pas disposés à apporter le soutien financier nécessaire à la matérialisation du NE$\mathrm{PAD}$, qui demure encore un éléphant blanc dans les multiples musées économiques africains. Tel est le deficit prospectif des fondateurs du NEPAD. Il est indispensable pour 
l'Afrique de trouver des sources alternatives et autonomes de financment des projets continentaux de développement industriel.

\section{Les Fonds continental pour le développement industriel et fonds régional d'investissement}

Il est nécessaire de consolider le plan de l'Union africaine visant à mettre en place un fonds panafricain chargé de financer le développement de l'industrie et de l'appareil de production ainsi que les projets d'infrastructure. Agissant sous la direction du NEPAD ou de la BAD et oeuvrant en étroite collaboration avec des institutions multilatérales de financement du développement telles que l'Agence multilatérale de garantie des investissements et la Banque internationale pour la reconstruction et le développement, le fonds dont la création est envisagée accélérerait la mobilisation des fonds sur les marchés des capitaux locaux et intérieurs, la collecte des contributions des gouvernements africains et l'acheminent de l'APD.

Les fonds régionaux d'investissement devraient faciliter l'élaboration des projets qui généreront à coup sûr des bénéfices. Même si les projets du secteur privé seront une priorité pour les fonds en question, les projets destinés à développer l'infrastructure qui ont une incidence directe sur la croissance de l'industrie seront également prioritaires.

\section{AIDA, cadre régional pour l'industrialisation de l'Afrique}

Pour être couronnés de succès, la politique et le développement industriels doivent être adaptés au contexte local dans le pays et coordonnés au niveau régional. Si la responsabilité du développement industriel incombe au premier chef aux gouvernements nationaux, l'intégration régionale a un rôle important à jouer s'agissant de lever les diverses restrictions à l'industrialisation. C'est ainsi que la coopération régionale au développement de l'infrastructure ferait baisser les coûts de transaction, renforcerait le développement des marchés régionaux et rendrait plus compétitives la production manufacturière et les exportations de produits manufacturés. L'intégration régionale peut également contribuer à réduire le fardeau réglementaire pesant sur les entreprises africaines en harmonisant par exemple les politiques et en servant d'organisme extérieur de modération en ce qui concerne les politiques intérieures. Elle stimulera aussi le commerce intra-africain et accélérera, par ricochet, l'industrialisation en Afrique. À cet égard, l'AIDA et sa Stratégie de mise en oeuvre offrent des possibilités pratiques de développement industriel accéléré aux niveaux régional et continental.

Les chefs d'État africains ont pris plusieurs décisions, en particulier dans le cadre de l'AIDA, en vue d'industrialiser le continent. Une série de 16 programmes et de 49 projets à mener au sein des sept modules de la "Stratégie de mise en oeuvre » de l'AIDA ont été définis. Les modules sont les suivants : élaboration de politiques industrielles et orientations stratégiques; renforcement des capacités productives et commerciales; développement des infrastructures et de l'énergie pour la transformation industrielle; développement des 
compétences techniques et industrielles; développement des systèmes d'innovation industrielle, de la recherche-développement et de la technologie; financement et mobilisation des ressources; et développement durable (document de l'Union africaine sur l'AIDA)

\section{Sociétés minières transnationales, responsabilité sociale et développement durable des peuples autochtones : sillon d'une lutte internationale pour le droit inaliénable}

Les peuples autochtones sont devenus de plus en plus visibles sur la scène internationale depuis notamment l'adoption de la Déclaration sur les Droits des Peuples Autochtones en septembre 2007. Ils développent leurs organisations et partenariats avec les organisations non gouvernementales (ONG), les agences spécialisées du systèmes des Nations Unies et les Organisations continentales (Union Européenne, Union Africaine, Organisation des États Américaines). Ils ont ainsi introduit des éléments de réflexion dans les mécanismes onusiens, notamment en surfant sur la vague du développement durable, du respect et de la protection de l'environnement, contexte valorisant l'idée que les peuples autochtones seraient détenteurs d'un savoir spécifique quant à la gestion de leur environnement naturel.

L'intérêt des institutions onusiennes se justifie par le fait que près de la moitié des peuples autochtones du monde vivent dans des zones menacées de destruction comme les forêts tropicales représentant à elles seules $80 \%$ de la biodiversité mondiale. Depuis la première « décennie internationale des peuples autochtones » (1995-2004), la mobilisation accrue autour des peuples autochtones est en partie liée aux négociations internationales sur l'environnement. Alors qu'auparavant c'était l'espace peu transformé et non habité qui était mis en avant, l'UNESCO avec le programme « Man and the Biosphere » (MAB) a entériné dès les années 1960, l'idée d'une conservation incluant des zones utilisées. En 1982, le Congrès de Bali voit l'émergence du concept de développement durable et en 1992 lors de la Conférence des Nations Unies sur l'environnement et le développement, les populations autochtones sont déclarées comme ayant « un rôle vital à jouer dans la gestion de l'environnement et le développement du fait de leurs connaissances du milieu et de leurs pratiques traditionnelles. Les États devraient reconnaître leur identité, leur culture et leurs intérêts, leur accorder tout l'appui nécessaire et leur permettre de participer efficacement à la réalisation d'un développement durable». Le Sommet mondial pour le développement durable a franchi une nouvelle et modeste étape en réaffirmant: « ... le caractère essentiel du rôle des populations autochtones dans le cadre du développement durable» ${ }^{55}$.

En effet, l'idéologie sous-jacente qui apparaît à travers ces différents sommets mondiaux, est celle d'une conservation biologique visant la sauvegarde de ce qui est utile et les années 1990 voient la valorisation de la biodiversité, souvent réduites à ses composantes potentiellement exploitables ${ }^{56}$. Les peuples autochtones surfant sur cette vague naturaliste

55 Voir la Déclaration de Johannesburg sur le développement durable, par. 25. Disponible en ligne sur ww.johannesburgsummit.org.

56 DEMMER, Ch., « Autochtonie, nickel et environnement : une nouvelle stratégie kanak », $\mathrm{Va}$ carme, $\mathrm{n}^{\circ} 39$, printemps 2007, pp. 43-48, spécialement p. 46. 
tendant à les faire participer, ont ainsi bénéficié d'un mouvement envers la protection de la biodiversité passant notamment par la Convention sur la diversité biologique (CDB), dont l'article $8 \mathrm{j}$ reconnaît explicitement, que grâce à leur mode de vie séculaire, ces peuples ont contribué à préserver la biodiversité des régions qu'ils habitent. Cet article a largement contribué à sortir la gestion des ressources naturelles du seul cadre écologique pour aborder des questions plus politiques, notamment la reconnaissance des groupes sociaux marginalisés et le partage des bénéfices liés à l'usage de ces ressources. La Convention 169 de l'Organisation Internationale du Travail sur les peuples indigènes et tribaux, de 1989, adoptée par 19 États dont 13 en Amérique du Sud, répondait déjà à ce souci à travers ses articles 14, 6 et 7 portant sur le droit à la terre et à la consultation ${ }^{57}$, mais aussi sur le droit de décider des priorités de développement. La Déclaration sur les droits des peuples autochtones a consacré ces questions à travers les articles 26, 28, 29, 30 et 32 portant sur les questions de gestion des terres, territoires et ressources. Ils concernent leurs droits sur les terres même en l'absence de titres de propriété octroyés par l'État, la reconnaissance de ce droit par les États et la protection des territoires concernés, le droit à la réparation, restitution ou indemnisation, à la préservation de l'environnement et de la capacité de production des terres ainsi qu'à l'atténuation des effets néfastes de la production sur les terres. Enfin, est reconnu le principe de consentement libre, préalable et connaissance de cause pour tous les projets concernant les terres et territoires où vivent des autochtones.

De ce qui précède, la Déclaration sur les droits des peuples autochtones peut apparaître comme la résultante d'une réflexion de vingt ans, non pas seulement menée dans le cadre du Groupe de Travail sur les Peuples Autochtones, mais plus largement au sein des Nations Unies, sur les implications de la croissance, la signification du développement pour les peuples restés en marge de cette dynamique.

Du côté des peuples autochtones, cette vague de préoccupations environnementales leur a permis de mettre le doigt sur des cas concrets, dont les multinationales minières sont emblématiques, par la conception même des sites d'exploitation (base-vie, isolation de la vie locale, voire militarisation des sites pour les protéger, conséquences environnementales particulièrement visibles). L'identification de ces problèmes spécifiques aux peuples autochtones concernant les industries extractives est apparue avant même l'adoption de la Déclaration des Droits des Peuples Autochtones. Ainsi, en 2003, alors que le Groupe de Travail de l'ONU sur les populations autochtones tenait une session spéciale portant sur le thème de la mondialisation, les peuples autochtones ont fait ressortir sa double caractéristique :

57 L'article 14 de la Convention stipule que non seulement : « (...) les droits de propriété et de possession des terres occupées " traditionnellement 》 doivent être reconnus mais également que des mesures doivent être prises pour sauvegarder des terres "non exclusivement occupées par eux, mais auxquelles ils ont traditionnellement accès pour leurs activités traditionnelles et de subsistance ». La Convention réserve également un rôle d'acteurs aux peuples indigènes dans le cadre de la planification et de la mise en oeuvre des projets de développement qui les affectent. Ces principes visent à garantir l'accès à l'information et à la prise de décision des peuples indigènes concernant toutes décisions qui affectent directement leurs conditions de vie. 
- son caractère pratique, les nouvelles technologies permettant d'échanger des idées et de développer des réseaux de solidarité;

- son caractère destructeur, assimilé à du néo-colonialisme, notamment du point de vue de l'environnement, de la non-régulation des activités des firmes transnationales, de l'absence de consultation et de protection des communautés locales pour qui les autochtones ne représentent aucun enjeu politique.

L'instance permanente pour les questions autochtones (IPQA) ainsi que le Rapporteur spécial sur les droits des peuples autochtones, soulignait déjà la nécessité de la participation politique des peuples autochtones et défendait l'idée d'un partenariat global entre les peuples autochtones, les États et les acteurs économiques, grâce aux mécanismes internationaux ${ }^{58}$. En décembre 2001, un premier atelier s'est tenu à Genève, sur « Les peuples autochtones, les sociétés privées travaillant dans les secteurs des ressources naturelles, de l'énergie et de l'extraction minière, et les droits de l'homme ${ }^{59}$. En janvier 2003, le Rapporteur spécial pour les questions autochtones d'alors, Rodolfo Stavenhagen rendait son rapport sur l'incidence des projets de développement d'envergure ou à grande échelle sur les droits de l'homme et les libertés fondamentales des peuples autochtones ${ }^{60}$. En 2004, Erice Irène Daes, Rapporteur spéciale sur « la souveraineté permanente des peuples autochtones sur les ressources naturelles », publiait également une étude dans ce ens. Depuis le séminaire d'experts de 2006 sur « les peuples autochtones, la souveraineté permanente sur les ressources naturelles et leur lien à la terre », tous les ans, une étude est réalisée sur les relations entre les industries extractives et les peuples autochtones soit par l'Instance permanente pour les questions autochtones, soit par le Haut Commissariat aux droits de l'Homme soit par le Rapporteur spécial sur les questions autochtones, James Anaya ${ }^{61}$. Ce sujet sera d'ailleurs au centre de son action jusqu'à la fin de son mandat (fin 2015). Au coeur de tous

58 BELLIER, I., «Identité globalisée, territoires contestés : les enjeux des peuples autochtones dans la constellation onusienne ", Autrepart n³8, 2006, p. 99-118, spécialement p. 100; " Le développement et les peuples autochtones : conflits de savoirs et enjeux de nouvelles pratiques politiques ", in GERONIMI, V. et alii, Savoirs et politiques de développement. Questions en débat à l'aube du XXİ̀ siècle, Paris, Karthala.

59 E/CN.4/Sub.2/AC.4/2002/3.

60 E/CN.4/2003/90.

61 Atelier sur les perspectives en matière de relations entre les peuples autochtones et les compagnies industrielles, 1-4 juillet 2007, Salekhard, disponible en ligne sur http://www.un.org/esa/socdev/unp fii/documents/workshop_PRIPIC_Report.pdf; Atelier international sur les sociétés exploitant les ressources naturelles, les peuples autochtones et les droits humains : définition d'un cadre de consultation, de partage des bénéfices et de règlement des différends, Moscou, 3-4 décembre 2008, A/HRC/EMRIP/2009/5, disponible en ligne sur http://www.ohchr.org/Documents/Issues/IPeoples/ Seminars/International_workshop_concept_paper.pdf; Séminaire d'experts internationaux sur les droits des peuples autochtones, la responsabilité sociale et les industries extractives, 2009, disponible en ligne sur http://www.un.org/esa/socdev/unpfii/en/EGM_IPCR.html;; Rapport de James Anaya sur les sociétés minières opérant en territoires autochtones, juillet 2010; Rapport de James Anaya sur la Responsabilité sociale des Entreprises et les droits des peuples autochtones, juillet 2011. 
ces rapports, les questions de consultation et de consentement, des retombées économiques, d'avoir des possibilités de choix de son mode de développement sont devenues récurrentes; et le cadre de la responsabilité sociale d'entreprise et du développement durable explicitement désigné comme devant devenir une obligation.

La problématique d'accaparement des terres et de groupes vulnérables : un aspect négligé de la RSE

L'accaparement des terres est une violation des droits de l'homme et peut frapper des personnes destituées de leurs terres, qui se retrouvent alors sans domicile ni moyen de subsistance. Il est nécessaire d'identifier les groupes vulnérables sur la chaîne de valeur afin de prévenir les incidences négatives sur les droits de l'homme, notamment celles découlant de l'accaparement des terres. Certains groupes sont particulièrement vulnérables. Selon la Banque mondiale ${ }^{62}$, des peuples autochtones ont été chassés de leurs terres par des sociétés d'huile de palme et de minerais dans certains pays africains en raison d'une mauvaise interaction avec les communautés locales (gérée de façon culturellement inappropriée quant aux méthodes, à la forme et à la langue employées), et à l'encontre des normes établies par la Banque mondiale pour les peuples autochtones. Les femmes sont les plus exposées aux expulsions, car elles ne disposent que très rarement de titres de propriété formels et sont souvent exclues des négociations. La Banque mondiale a même suspendu une fois tout prêt à l'industrie de l'huile de palme afin de mettre en oeuvre de nouvelles stratégies préventives ${ }^{63}$.

Le Comité de la sécurité alimentaire mondiale (CSA) des Nations unies a adopté les Directives volontaires pour une gouvernance responsable des régimes fonciers, qui constituent le premier instrument juridique sur les questions foncières. Ces directives sont le fruit d'un long processus multipartite impliquant les États, les principales organisations internationales, la société civile et le secteur privé. Basées sur les droits de l'homme, elles incluent plusieurs principes et dispositions s'adressant au secteur privé, dans la veine des principes directeurs des Nations unies. Elles peuvent et doivent être appliquées à toutes les parties prenantes, y compris le secteur privé.

62 Banque mondiale, «Papua New Guinea: Smallholder Agriculture Development », 2009, disponible en ligne sur http://www.banquemondiale.org/projects/P079140/png-smallholder-agriculturev elopment?lang=fr, consulté le 10 janvier 2015.

63 GEARY, K., « Notre terre, notre vie : Halte à la ruée mondiale sur les terres », 2012, disponible en ligne sur http://policy-practice.oxfam.org.uk/publications/our-land-our-lives-time-out-on-the-globa 1-land-rush-246731, consulté le 5 janvier 2015. p. 8. 
Nécessité des normes internationales imposant la valeur ajoutée par la transformation des matières premières sur les territoires des pays producteurs et les contrôles intergouvernementaux des prix

L'abondance des richesses et la demande mondiale croissante de matières premières, poussent les gouvernements africains à établir actuellement de nouveaux partenariats, à s'emploier à accroitre les investissements dans les infrastructures et a acquérir du savoirfaire et de la technologie. Cependant, l'Afrique peut mieux faire : la production et l'exportation des matières premières à l'état brut équivalent à un abandon de recettes énormes du fait de la non-création de valeur ajoutée, à l'exportation d'emplois vers les pays qui peuvent ajouter de la valeur aux produits et à l'exposition à de hauts risques inhérents à la dépendance à l'égard des produits non renouvelables et des fluctuations de la demande et des prix des produits de base. Plutôt que de compter sur les exportations de matières premières, le continent africain devrait ajouter de la valeur à ses produits de base afin de promouvoir une croissance soutenue, la création d'emplois et la transformation économique. Dans cet engrenage, des pratiques de corruption au sommet de l'Etat devraient être bannies : il va de soi que des dirigeants dont les élections sont financées par des sociétés multinationales capitalistes, ne se rebifferont à conclure des contrats léonins ou avec ces derniers, galvaudant ainsi les matières premières. Dans certains cas, des législations nationales dans le domaine de production des matières premières sont monnayées et portent des clauses accordant des avantages exorbitants aux multinationales au détriment de communautés propriétaires. De même, des accords bilatéraux déséquilibrés désavantageant les peuples autochtones et sont conclus en contrepartie des appuis diplomatiques, militaires et divers d'autres Etats. Des institutions démocratiques fortes jonchées des mécanismes transparents de contrôle populaires, peuvent s'avérer une des solutions contre le bradage des matières premières. Dans une approche différente dans le cadre d'une étude ultérieure, l'on s'emploiera à démontrer que tel vœu doit être exaucé par les néo-libéralistes qui dirigent la planète!

En plus d'offrir des avantages comparatifs à moyen et à long terme, l'industrialisation fondée sur les produits de base peut, avec des politiques industrielles pertinentes, servir de tremplin pour une diversification à long terme et la compétitivité dans de nouveaux secteurs autres que ceux des matières premières dans les pays africains riches en produits de base $\mathrm{e}^{64}$.

Si les économies africaines exportatrices de produits de base ont grandement tiré avantage des récentes hausses soutenues des prix de leurs exportations de produits primaires et d'une augmentation des rentes tirées des ressources fiscales, on ne saurait cependant compter sur ces rentes pour jouer le rôle de moteur de la croissance et du développement. La raison est que non seulement les produits de base sont non renouvelables, mais aussi que la création de valeur ajoutée aiderait les pays africains à réduire leur exposition aux risques de fluctuation des cours de ces produits et en même temps passer à des produits à plus forte

64 Voir CEA et UA, Tirer le plus grand profit des produits de base africains...op.cit. 
valeur et plus diversifiés et à des marchés finaux sur lesquels les prix dépendent plus des fondamentaux du marché que de la spéculation.

En effet, l'intervention des agents financiers sur les marchés et la financiarisation du commerce des produits de base ont fréquemment conduit ces marchés à passer d'un cadre ou l'on vend au prix du jour à un cadre soumis aux rapports de force sur le marché, en partie parce qu'ils sont fortement concentrés et souvent caractérisés par une asymétrie de l'information. Les agents financiers sont devenus des acteurs clefs dans l'orientation de la spéculation et du comportement moutonnier et ont crée des distorsions sur les marchés des produits de base, avec notamment une tendance haussière des cours du café et du cacao et des prix bas sans précédent pour le coton.

Cette attitude a rendu les pays africains plus vulnérables aux fluctuations sur les marchés de produits de base, tandis que les prix artificiellement élevés de certains produits de base réduisent l'incitation à la création de la valeur ajoutée. La promotion d'une industrialisation fondée sur les produits de base pourrait offrir un puissant outil aux pays africains pour s'attaquer à cette « tyrannie de la financiarisation ${ }^{65}$ ». De même, l'exportation à l'état brut de nombre de matières premières constitue une activité à forte intensité de capital, qui empêche la création d'emplois et la distribution des rentes tirées de leur exploitation. Une autre piste de solution se trouve dans les accords interétatiques ou intergouvernementaux (FMI, BM) à travers lesquels les Etats peuvent exercer des contraintes sur des agents financiers et multinationales en vue de résorber le problème de spéculation de prix des matières premières dans les marchés internationaux et émettre des notes sur des perspectives de croissance de leurs économies, domaine jusque-là réservé aux agences de notation; c'est ce que s'emploi à faire l'Union européenne. Pareillement, l'initiative d'un Fond monétaire africain s'inscrit dans cette vue.

\section{Conclusion}

L'analyse de quelques normes internationales qui encadrent l'industrie des matières premières appelle deux observations : primo, les règles juridiques régissant la création d'une industrie multinationale des matières premières sont fixées conformément aux législations internes des Etats s'agissant des procédures d'installation, de fonctionnement, de droits et devoirs à l'égard de pouvoirs publics et communautés de base. Ceci implique notamment le respect des droits de l'homme, particulièrement des normes du travail, de normes environnementales et de lutte contre la corruption, cette dernière gangrenant sérieusement le trésor public en ce que les redevances qui lui sont dues échappent à son contrôle. De plus, ces droits et devoirs sont essentiellement consacrés dans les différents instruments facultatifs sus-évoqués sous forme de code de conduite, recommandations de bonnes pratiques en matière d'exploitation des matières premières, conceptualisés en responsabilité sociale d'entreprise. Ces normes se trouvent par ailleurs consignées dans le pacte international relatif 
aux droits sociaux et économiques....; secundo, ces recommandations ne permettent pas de forer la problématique de fond, au cœur de la lutte de la décennie septante menée par les pays du tiers-monde, dont l'Algérie a servi de fer de lance. Cette lutte avait abouti à l'adoption du nouvel ordre économique matérialisé par la Charte d'Alger, adoptée par la Conférence des Non-Alignés en 1973, la Charte des Droits et Devoirs économiques des Etats, adoptée par l'Assemblée générale le 12 décembre 1974, la Déclaration et le Plan d'action concernant le développement et la coopération industrielle, établis par les septante-sept Etats du tiers-monde en janvier 1975 : le commerce équitable entre les nations dont le leitmotiv demeure les matières premières doivent profiter au premier plan aux propriétaires terriens.

Cependant, le capitalisme joue un rôle essentiel dans le déséquilibre du commerce international en privilégiant les intérêts privés au détriment de communautés propriétaires. La rengaine du propos introductif de l'étude vaut : les multinationales avec leurs agents financiers, adeptes du néo-libéralisme, détiennent le pouvoir de décision sur les prix des matières premières, influencent les termes des accords internationaux et font du chantage lorsque les propriétaires des richesses décident des mesures protectionnistes. Pris en étau par le système financier et économique international, les pays africains devraient davantage consolider le commerce intra-régional et promouvoir des mécanismes financiers et commerciaux de portée régionale en vue de booster l'offre et la demande intra-africaine, de maitriser certains phénomènes financiers, souvent à la base de la spéculation et de fluctuations économiques. Ceci permettra d'influer sur les retombées en termes de recettes pour le trésor public, d'amélioration des conditions de vie des peuples autochtones, propriétaires des matières premières (infrastructures publiques, santé, éducation, emploi...) et de protection de leur environnement.

Il est ainsi nécessaire de développer une politique industrielle qui aide les pays africains à tirer profit de leurs avantages comparatifs pour être compétitifs, même au niveau des entreprises, sur le marché international. La stabilité macro-économique et des taux de change compétitifs en termes réels, voie empruntée par nombre d'Etats africains, sont des préalables indispensables pour obtenir des meilleurs résultats industriels, mais ne sont pas en soi des conditions suffisantes. Une condition particulièrement urgente en Afrique est d'investir dans le développement et la modernisation des infrastructures, si l'on veut que les entreprises réagissent à la dévaluation et à d'autres incitations par les prix. Les investissements publics dans ce domaine peuvent inciter le secteur privé à investir.

L'on peut s'accorder avec Smail Khennas ${ }^{66}$ que la question n'est plus celle de la place du secteur privé dans l'économie nationale mais celle de la nature du pouvoir et de son corollaire, qui est celui des orientations du mode de gestion des entreprises publiques. La thèse accréditant l'entreprise publique comme la source de l'échec tend à substituer la cause aux effets. La question soulevée par l'auteur parait pertinente : "dans quel pays africain, un capital privé dynamique disposant d'une surface financière considérable, d'une accu- 
mulation technologique et d'une capacité organisationnelle est-il en mesure de répondre aux nouveaux défis nationaux et internationaux ${ }^{67}$ ?

Même si certains thinks tanks capitalistes considèrent que la globalisation et la transnationalisation industrielles tendent à minimiser le rôle de l'Etat en matière économique, paradigme à relativiser dans le contexte africain, les abus des multinationales prenant des proportions plus inquiétantes, les prérogatives de puissance publique demeurent indispensables pour une protection des droits de l'homme et des peuples. Elles garantissent le progrès collectif notamment par la conclusion des accords bi et multilatéraux qui tiennent véritablement compte du développement durable des peuples africains. Ceci requiert, pour les dirigeants africains, de se munir de l'antidote du néo-libéralisme sauvage.

\section{Références bibliographiques}

Banque mondiale, « Papua New Guinea: Smallholder Agriculture Development », 2009, disponible en ligne sur http://www.banquemondiale.org/projects/P079140/png-smallholder-agriculturevelopmen t?lang=fr, consulté le 10 janvier 2015.

Bureau du pacte mondial des Nations Unies, L'entreprise citoyenne dans l'économie mondiale. Le Pacte Mondial des Nations Unies, disponible en ligne surhttp://www.unglobalcompact.org/langua ges/french/index.html. consulté le 13 décembre 2014.

BELLIER, I., «Identité globalisée, territoires contestés : les enjeux des peuples autochtones dans la constellation onusienne », Autrepart n`38, 2006.

BELLIER, I., « Le développement et les peuples autochtones : conflits de savoirs et enjeux de nouvelles pratiques politiques », in GERONIMI, V. et alii, Savoirs et politiques de développement. Questions en débat à l'aube du XXİ̀ siècle, Paris, Karthala, 2012.

CAMPBELL, B., Étude sur le rôle du secteur privé dans la réalisation des intérêts du Canada en développement international, Le Comité permanent des affaires étrangères et du développement international, 4 avril, 2012.

CEA et UA, Tirer le plus grand profit des produits de base africains: l'industrialisation au service de la croissance, de l'emploi et de la transformation économique, Rapport économique sur l'A frique 2013.

CEA et UA, L'industrialisation au service de l'émergence de l'Afrique, Document de synthèse de la Sixième réunion conjointe de la Conférence des ministres africains des finances, de la planification et du développement économique de la Commission économique pour l'Afrique et de la Conférence des ministres de l'économie et des finances de l'Union africaine, Abidjan (Côte d'Ivoire) 25 et 26 mars 2013.

CETIM, «Les sociétés transnationales, acteurs majeurs dans les violations des Droits de l'Homme », Cahier critique ${ }^{\circ} 10$, décembre 2011.

CNUCED,Statistics:UNCTAD.org., 2012, disponible en ligne sur https://unctadstat.unctad.org/TableVi ewer/tableview.aspx?Reportid=88, consulté le 18 janvier 2015.

67 Ibidem. 
Industrie des matières premières et responsabilité sociale d'entreprise en Afrique : réflexion sur le cadre juridique international

Charte d'Alger, adoptée par la Conférence des Non-Alignés en 1973.

Charte des Droits et Devoirs économiques des Etats, adoptée par l'Assemblée générale le 12 décembre 1974.

Convention de Vienne sur le droit des traités du 23 mai 1969. Disponible sur https://textesdipannotes.files.wordpress.com/2011/07/c-v-19691.pdf. consulté le 10 aout 2015.

DAUGAREILH, I., Responsabilités de l'entreprise transnationale et globalisation de l'économie, Paris-Bruxelles, Bruylant-LGDJ, 2010.

Déclaration et de Plan d'action concernant le développement et la coopération industrielle, adoptés en janvier 1975.

DEMMER, Ch., «Autochtonie, nickel et environnement : une nouvelle stratégie kanak », Vacarme, n ${ }^{\circ} 39$, printemps 2007.

DOGBEVI, E., What Carlos Lopes said at the start of ADF VIII, ADF TODAY, 25 octobre, 2012.

DOGBEVI, E, African Mining Vision not widely known Graham, ADF TODAY, 25 octobre, 2012.

GEARY, K., « Notre terre, notre vie : Halte à la ruée mondiale sur les terres », 2012.

ILO, « Forced Labour Mechanism », disponible en ligne surhttp://www.ilo.org/yangon/complaints/lang -en/index.htm. Consulté le 19 novembre 2014.

Le Haut Commissariat des Nations Unies aux droits de l'homme, le Programme des Nations Unies pour l'environnement, l'Organisation internationale du travail (OIT) et le Programme des Nations Unies pour le développement.

Le Haut Commissariat des Nations Unies aux droits de l'homme, Principes directeurs relatifs aux entreprises et aux droits de l'homme : mise en ouvre du cadre de référence " protéger, respecter et réparer» des Nations Unies, New York et Genève, 2011.

LEVACHER, C., Les sociétés transnationales minières face au droit des peuples autochtones. Quels acteurs, pour quels enjeux?, GITPA, Septembre 2012.

MARECHAL, «La dimension sociale du développement durable », Alternatives Economiques, n¹91, avril 2001.

MAZUYER, J., L'application du Pacte mondial des Nations Unies par les entreprises françaises : un exemple de « bonnes pratiques » de RSE?, 2012.

$\mathrm{OCDE}$, Les principes directeurs de l'OCDE à l'intention des entreprises multinationales, Editions OCDE, 2011, disponible en ligne sur http://www.oecd.org/fr/daf/inv/mne/48004355.pdf, consulté le 20 novembre 2015.

OIT, Déclaration de principes tripartite sur les entreprises multinationales et la politique sociale, adoptée par le Conseil d'administration du Bureau international du Travail à sa $204^{\mathrm{e}}$ session (Genève, novembre 1977), telle qu'amendée par le Conseil à sa $279^{\mathrm{e}}$ session (Genève, novembre 2000) et à sa $295^{\mathrm{e}}$ session du 28 mars 2006, disponible en ligne http://www.ilo.org/wcmsp5/groups /public/_ed_emp/_mp_ent/documents/publication/wcms_124923.pdf, consulté le 10 novembre 2014.

OXFAM, " Oxfam International's Perspective on the Draft Guiding Principles for the mplementation of the United Nations "Protect, Respect and Remedy" Framework », 2011, disponible en ligne sur http://www.oxfamamerica.org/files/oxfam-ruggie-guiding-principles.pdf, consulté le 10 février 2015. 
Pacte Mondial Réseau France, http://www.pactemondial.org/, consulté le 10 janvier 2015.

SMAIL KHENNAS, " Préface ", in SMAIL KHENNAS (dir.), Industrialisation, ressources minières et énergie en Afrique, Dakar, CODESRIA, 1993.

TATOU, R., Europe outre-mer, avril 1974, n 531.

TOUSCOZ, J., « La coopération internationale et les matières premières exportées par les pays du Tiers Monde : les conditions d'un échange égal ", Tiers-monde, 1976, tome $17 \mathrm{n}^{\circ} 66$, les produits de base et la politique internationale, pp. 539-560, disponible en ligne sur http://www.persee.fr/we b/revue/home/prescript/article/tiers_0040-7356_1976_num_17_66_2648, consulté le 14 septembre 2014. 